\title{
Improved ground state rotational constants for the argon-hydrogen fluoride dimer
}

\author{
Paul A. Stockman \\ Division of Chemistry and Chemical Engineering, California Institute of Technology 170-25, \\ Pasadena, California 91125 \\ Geoffrey A. Blake \\ Division of Geological and Planetary Sciences, California Institute of Technology 170-25, \\ Pasadena, California 91125
}

(Received 20 October 1992; accepted 12 November 1992)

In the past decade, the intermolecular potential of the argon-hydrogen fluoride dimer (Ar-HF) has been probed by microwave spectroscopy of the vibrational ground state $^{1-3}$ and by intramolecular ${ }^{4-7}$ and intermolecular ${ }^{5-9}$ spectra of the HF fundamental stretch and van der Waals modes. Also, the intermolecular potential energy surface (IPS) of the Ar-HF molecule has been the focus of several theoretical ${ }^{10,11}$ studies. While the ground state has been probed directly by microwave spectroscopy only to the $J=3$ level, subsequent infrared studies have measured transitions arising from the ground vibrational state with rotational energies up to $J=40$, albeit with a nearly thousandfold decrease in experimental precision. Analyses of these infrared spectra have required Hamiltonians containing high-order centrifugal distortion constants due to large amounts of anharmonicity in the high- $J$ rotationally predissociative states. ${ }^{5}$

As experimental and theoretical studies probe ever higher regions of the IPS, the need for improved ground state rotational constants becomes apparent. Indeed, a number of new experimental techniques have recently been developed to probe the overtone spectra of clusters for which the availability of accurate ground state term values would greatly aid in the assignment and fitting of the data. ${ }^{9,12}$ We therefore present here extended microwave and millimeter-wave measurements of the ground vibrational state rotational transitions up to $J(13 \leftarrow 12)$. A combined weighted fit with previously measured rotational transitions ${ }^{1(b)}$ and high- $J$ infrared spectra $\left(10^{\circ} 0\right) \leftarrow\left(00^{0} 0\right)$ (excitation of the HF monomer) ${ }^{5}$ is used to extract an expanded set of ground state rotational constants with improved precision and accuracy. The resulting term values have proven useful in fits of the third overtone spectra of $\mathrm{Ar}-\mathrm{HF}$ obtained by Chang and Klemperer. ${ }^{12}$

The experimental apparatus employed is the same as that previously described, ${ }^{13}$ consisting of microwaves broadcast and received by tapered waveguide horns across the supersonic expansion through a 1.75 in. $\times 0.001$ in. slit. The effective cutoff of the spectrometer after applying a series of frequency doublers and triplers to the 0.01 to 20 $\mathrm{GHz}$ source was $\sim 80 \mathrm{GHz}$. The control of the gases was also as before, using an expansion of $5.80 \mathrm{slm} \mathrm{Ar}$ and 120 sccm HF. Automated scans of the observed rotational transitions with both increasing and decreasing frequency increments were taken and averaged to eliminate errors due to the time constant of the lock-in amplifier. Rotational transitions from $J(4 \leftarrow 3)$ to $J(13 \leftarrow 12)$ were recorded. These are presented in Table I, along with the lowest three rotational transitions previously observed.

The data were fit as in previous efforts ${ }^{5,7}$ to a power series in $J(J+1)$ :

$$
\begin{aligned}
E(v, J)= & v+B_{v} J(J+1)-D_{v} J^{2}(J+1)^{2}+H_{v} J^{3}(J+1)^{3} \\
& +L_{v} J^{4}(J+1)^{4}+P_{v} J^{5}(J+1)^{5}
\end{aligned}
$$

with a nonlinear least-squares analysis. The rotational constants from Dixon $e t a l .^{1(b)}$ were used to generate the three lowest transition frequencies free from the effects of spinspin interactions. As the emphasis throughout was on obtaining the most accurate rotational constant values possible for the ground state, care was taken not to affect the lower order constants with the large anharmonicity exhibited by the high- $J$ infrared spectra. Consequently, the microwave transitions were fit with constants up to $L_{0}$, with the data from Dixon et al. being weighted with $\sigma \approx 5 \mathrm{kHz}$ and the present frequencies having an estimated error of $\sigma \approx 100 \mathrm{kHz}$, in accordance with the measured precision of the two data sets. $B_{0}$ and $D_{0}$ were fixed at values from this fit in the combined analysis of the microwave and infrared data and the value of $H_{0}$ was made 1000 times more resistant than the other determined parameters. The infrared data set was also weighted with respect to its measured precision, with most lines having a weight of $\sigma \approx 20 \mathrm{MHz}$. A few lines corresponding to blends in the $P$-branch head and transitions near interfering HF dimer lines ${ }^{14}$ were assigned larger estimated errors. The results of this fit are presented in Table II with rotational constants for both the ground, $v(\mathrm{HF})=0$, and $\left(10^{\circ} 0\right), v(\mathrm{HF})=1$, vibrational states.

Considering first the results from the fit of the microwave data sets in Table I, a comparison of the present rotational constants with those from previous high resolution studies of the lowest few transition frequencies reveals no discrepancy in the $B_{0}$ values within $\pm \sigma$. The first distortion term $D_{0}$ is approximately $1 \%$ smaller than earlier reported values, having been partially freed from the effects of higher-order distortion by a well-determined $H_{0}$ value, while at the same time benefiting from the contributions of higher- $J$ transitions. Constants through $L_{0}$ were necessary 
TABLE I. Measured microwave transition frequencies (MIIz) and fitted rotational constants (MHz) for Ar-HF.

\begin{tabular}{|c|c|c|c|}
\hline$J^{\prime \prime}$ & $J^{\prime}$ & Measured & Kesidual $^{\mathrm{a}}$ \\
\hline 0 & 1 & $6131.132^{b}$ & 0.002 \\
\hline 1 & 2 & $12260.565^{b}$ & 0.000 \\
\hline 2 & 3 & $18386.601^{b}$ & -0.001 \\
\hline 3 & 4 & 24507.58 & 0.05 \\
\hline 4 & 5 & 30621.66 & 0.05 \\
\hline 5 & 6 & 36727.10 & 0.02 \\
\hline 6 & 7 & 42822.16 & -0.01 \\
\hline 7 & 8 & 48905.04 & -0.02 \\
\hline 8 & 9 & 54973.88 & -0.01 \\
\hline 9 & 10 & 61026.82 & 0.06 \\
\hline 10 & 11 & 67061.70 & -0.04 \\
\hline 11 & 12 & 73076.87 & -0.01 \\
\hline 12 & 13 & 79070.17 & 0.01 \\
\hline Constants & & Fitted Values & \\
\hline$B_{0}$ & & $3065.7063(43)$ & \\
\hline$D_{0}$ & & $0.70578(89) \times 10^{-1}$ & \\
\hline$H_{0}$ & & $-0.894(69) \times 10^{-5}$ & \\
\hline$L_{0}$ & & $0.40(18) \times 10^{-8}$ & \\
\hline
\end{tabular}

${ }^{a}$ Residual = observed - calculated.

${ }^{b}$ Generated from constants of Ref. 1(b); see text.

in order to bring the fit to the level of experimental uncertainty.

The global fit combining both microwave data sets and the $\left(10^{0} 0\right) \leftarrow\left(00^{0} 0\right)$ infrared data of Fraser and Pine ${ }^{5}$ is essentially the splicing of two polynomial functions. The preferential weighting of the microwave data ensures that the pure rotational frequencies contribute more to the determination of the lower-order ground state terms and the high- $J$ infrared data weigh more heavily in the determination of the two highest-order ground vibrational state terms $L_{0}$ and $P_{0}$. While significantly different from those determined by Fraser and Pine, the set of constants given in Tablc II arc qualitativcly similar in magnitude and sign as expected. However, the same comparison cannot be made with the constants obtained by Lovejoy and Nesbitt. ${ }^{7}$

TABLE II. Globally fitted rotational constants (MHz) for $\Lambda \mathrm{r}-\mathrm{HF}$.

\begin{tabular}{|c|c|c|}
\hline & $v=0$ & $v=1$ \\
\hline$B$ & $3065.7063^{a}$ & $3076.84(23)$ \\
\hline$D$ & $0.70578 \times 10^{-1 \mathrm{a}}$ & $0.6518(90) \times 10^{-1}$ \\
\hline$H$ & $-0.852(51) \times 10^{-5}$ & $-0.30(14) \times 10^{-5}$ \\
\hline$L$ & $0.228(66) \times 10^{-8}$ & $-0.171(95) \times 10^{-8}$ \\
\hline$P$ & $-0.254(22) \times 10^{-11}$ & $-0.62(24) \times 10^{-12}$ \\
\hline$E_{v}$ & \multicolumn{2}{|c|}{$3951.76842(55) \mathrm{cm}^{-1}$} \\
\hline
\end{tabular}

${ }^{\mathrm{a}}$ Fixed at value from Table I.
Their observation of the rovibrational $\left(10^{\circ} 0\right) \leftarrow\left(00^{\circ} 0\right)$ band in a $\sim 13 \mathrm{~K}$ beam limited them to measure transitions from $J \leqslant 27$. Both their data set and ours are insensitive to the higher-order distortion seen in the $211 \mathrm{~K}$ static White cell experiment of Fraser and Pine. Consequently, the ground state constants of Lovejoy and Nesbitt place the pure rotational band head for Ar-HF at $J(90 \leftarrow 91)$ as opposed to $J(60 \leftarrow 61)$ for Fraser and Pine or $J(58 \leftarrow 59)$ for the present work.

It is also interesting to note an increase in the value of the $B$ rotational constant from the ground state to the $\left(10^{\circ} 0\right)$ state along with a uniform decrease in the magnitudes of the associated distortion constants. This is consistent with the findings of Huang et al. that the first excited state of the HF monomer within Ar-HF exhibits a remarkably long vibrational predissociative lifetime $(\tau \geqslant 300 \mu \mathrm{s})$ due to a shortening and stiffening of the van der Waals bond.

In conclusion, we have extended considerably the ground state microwave spectra of Ar-HF. These new transition frequencies in turn have allowed us to improve upon the existing rotational constants for the ground state by a combined fit with high- $J$ infrared data. The errors in the term values are under $1 \mathrm{MHz}$ up to $J=20$, and $\leqslant 30$ $\mathrm{MHz}$ for $J$ values up to 40 .

This research was supported by NSF Grant No. CHEM-8957228. We thank W. Klemperer for suggesting this work and G. T. Fraser for suppiying us with the data from Ref. 5. We also gratefully acknowledge partial financial support from the David and Lucille Packard and Alfred P. Sloan Foundations as well as the support and services rendered by the Caltech staff. Division of Geological and Planetary Sciences, California Institute of Technology, Contribution No. 5219.

${ }^{1}$ (a). S. J. Harris, S. E. Novick, and W. Klemperer, J. Chem. Phys. 60, 3208 (1974); (b) A. Dixon, C. H. Joyner, F. A. Baiocchi, and W. Klemperer, ibid. 74, 6539 (1981).

${ }^{2}$ M. R. Keenan, L. W. Buxton, E. J. Campbell, A. C. Legon, and W. H. Flygare, J. Chem. Phys. 74, 2133 (1981).

${ }^{3}$ B. L. Cousins, S. C. O'Brien, and J. M. Lisy, J. Phys. Chem. 88, 5142 (1984).

${ }^{4}$ M. A. Dvorak, S. W. Reeve, W. A. Burms, A. Grushow, and K. R. Leopold, Chem. Phys. Lett. 185, 399 (1991).

${ }^{5}$ G. T. Fraser and A. S. Pine, J. Chem. Phys. 85, 2502 (1986).

${ }^{6}$ C. M. Lovejoy, M. D. Schuder, and D. J. Nesbitt, J. Chem. Phys. 85, 4890 (1986).

${ }^{7}$ C. M. Lovejoy and D. J. Nesbitt, J. Chem. Phys. 91, 2790 (1989).

${ }^{8}$ Z. S. Huang, K. W. Jucks, and R. E. Miller, J. Chem. Phys. 85, 6905 (1986).

${ }^{9}$ A. McIlroy and D. J. Nesbitt, Chem. Phys. Lett. 187, 215 (1991).

${ }^{10}$ F. R. Burden and A. Cuna, J. Mol. Struct. 189, 25 (1988).

${ }^{11}$ J. M. Hutson, J. Chem. Phys. 96, 6752 (1992).

${ }^{12}$ H. C. Chang and W. Klemperer, J. Chem. Phys. (submitted).

${ }^{13}$ R. E. Bumgarner and G. A. Blake, Chem. Phys. Lett. 161, 308 (1989).

${ }^{14}$ G. T. Fraser (private communication). 